\title{
AN APPLICATION OF A THEOREM OF SHEILA BRENNER FOR HOCHSCHILD EXTENSION ALGEBRAS OF A TRUNCATED QUIVER ALGEBRA
}

\author{
HIDEYUKI KOIE
}

\begin{abstract}
Let $A$ be a truncated quiver algebra over an algebraically closed field such that any oriented cycle in the ordinary quiver of $A$ is zero in $A$. We give the number of the indecomposable direct summands of the middle term of an almost split sequence for a class of Hochschild extension algebras of $A$ by the standard duality module $D(A)$.
\end{abstract}

\section{INTRODUCTION}

Brenner [2] studied the number of indecomposable direct summands of the middle term of an almost split sequence and she showed how to determine this number for an artin algebra. However, it is not easy to compute this number by original method. Fernández-Platzeck [3] obtained further information for trivial extension algebras. Similarly, we will give an application of a theorem of Sheila Brenner for Hochschild extension algebras which is a generalization of trivial extension algebras.

Let $K$ be an algebraically closed field and $A=K \Delta_{A} / I$ a bound quiver algebra, where $\Delta_{A}$ is a finite connected quiver and the ideal $I$ is admissible. We denote by $D(A)$ the standard duality module $\operatorname{Hom}_{K}(A, K)$. We recall the definitions of a Hochschild extension and a Hochschild extension algebra from [4], [5] and [10]. By a Hochschild extension over $A$ by $D(A)$, we mean an exact sequence

$$
0 \longrightarrow D(A) \stackrel{\kappa}{\longrightarrow} T \stackrel{\rho}{\longrightarrow} A \longrightarrow 0
$$

such that $T$ is a $K$-algebra, $\rho$ is an algebra epimorphism and $\kappa$ is a $T$-bimodule monomorphism. The algebra $T$ is called a Hochschild extension algebra. It is well known that $T$ is isomorphic to $A \oplus D(A)$ with the multiplication

$$
(a, f)(b, g)=(a b, a g+f b+\alpha(a, b)),
$$

where $\alpha: A \times A \longrightarrow D(A)$ is a 2-cocycle. We denote by $T_{\alpha}(A)$ the Hochschild extension algebra corresponding to a 2-cocycle $\alpha$. Then, $T_{0}(A)$ is just the trivial extension algebra $A \ltimes D(A)$.

For a hereditary algebra $A$, Yamagata [9] studied the Auslander-Reiten quivers of Hochschild extension algebras. In particular, for a Hochschild extension algebra $T$, he showed that the number of isomorphism classes of indecomposable modules in $\bmod T$ is twice the one for $\bmod A$. However this does not hold for a Hochschild extension algebra for a general algebra. So we are interested in an almost split sequence in the module category of Hochschild extension algebra.

2010 Mathematics Subject Classification. 16E40, 16G20, 16G70.

Key words and phrases. Hochschild extension, Hochschild (co)homology, trivial extension, selfinjective algebra, almost split sequence, quiver. 
In [2], Brenner showed how to determine the number of indecomposable direct summands of the middle term of an almost split sequence starting with a simple module. As a consequence of this result, for a self-injective artin algebra, she obtained the number of indecomposable direct summands of $\operatorname{rad} P / \operatorname{soc} P$, where $P$ is an indecomposable projective module. These results by Brenner play an important role in the representation theory of algebras. However, in general, it is not easy to compute these numbers for a given algebra. So there is few works to compute these numbers. In [3], Fernández and Platzeck gave a simple interpretation of them in the particular case of the trivial extension $T_{0}(A)$. This is done by focusing on the number of nonzero cycles in $\Delta_{T_{0}(A)}$. Fernández and Platzeck proved that the set of nonzero cycles coincides with the set of elementary cycles. Using this fact, they gave the numbers considered by Brenner by computing the cardinality of the equivalent classes of the set of nonzero cycles.

In this paper, for a truncated quiver algebra $A$ such that any oriented cycle is zero in $A$, we give a similar interpretation of the numbers considered by Brenner for a Hochschild extension algebra $T_{\alpha}(A)$ such that $\Delta_{T_{\alpha}(A)}=\Delta_{T_{0}(A)}$ holds. Unfortunately, for a Hochschild extension algebra, the set of nonzero cycles does not coincide with the set of elementary cycles in general. So by defining an $\alpha$-revived cycle, we will prove that a nonzero cycle in $T_{\alpha}(A)$ is either an elementary cycle or an $\alpha$-revived cycle. So we enumerate these nonzero cycles and then we can give the numbers considered by Brenner easily.

This paper is organized as follows: In Section 2, we recall results of Brenner, some definitions and facts about Hochschild extension algebras. In particular, some facts about 2-cocycles and the ordinary quiver of Hochschild extension algebras are based on [5] and [6]. In Section 3, we will give a characterization of nonzero oriented cycles in a Hochschild extension algebra. We will show that a nonzero oriented cycle in $T_{\alpha}(A)$ is either an elementary cycle or an $\alpha$-revived cycle. In Section 4 , we give the number of indecomposable direct summands of the middle term of almost split sequence for $T_{\alpha}(A)$. We define a relation in the set of all nonzero cycles with an origin $h$ in $T_{\alpha}(A)$. And we give the number by computing the cardinality of the set of equivalence classes. Moreover, for a class of Hochschild extension algebra, we will show that if the origin $h$ is neither sink nor source then the number is one.

For general facts on quivers and bound quiver algebras, we refer to [1] and [8]. Also for Hochschild extension algebra, we refer to [5], [6], [9] and [10]. Moreover, the notation including $\Delta_{0}, \Delta_{1}, \Delta_{+}$and the isomorphism $\Theta: \bigoplus_{q} D\left(H H_{2, q}(A)\right) \stackrel{\sim}{\longrightarrow}$ $H^{2}(A, D(A))$ is same as in $[5]$.

\section{Preliminaries}

In this section, we recall theorems of Brenner [2], the ordinary quiver of a Hochschild extension algebra along [5] and the definition of an elementary cycle in the ordinary quiver of a Hochschild extension algebra introduced in [3]. After that we define an $\alpha$-revived cycle for a 2-cocycle $\alpha$ and we give an example of these cycles.

2.1. A theorem of Brenner. We recall Brenner's results along her paper [2]. Let $A$ be an artin algebra. An element of $A$ of the form $a=x a y$ will be called an arrow, where $x$ and $y$ are primitive idempotents of $A$ and $a \in \operatorname{rad} A \backslash \operatorname{rad}^{2} A$. Let $e$ 
be a primitive idempotent of $A$. A set $\Lambda$ of arrows will be called a complete set of arrows for $\operatorname{rad} A e$ if

- it generates rad $A e$ as a left $A$-module,

- no proper subset of $\Lambda$ generates $\operatorname{rad} A e$.

A complete set of arrows for $\operatorname{rad} e A$ is defined similarly.

Let $e$ be a primitive idempotent. We denote by $\mathscr{N}$ the set of pair $(N, n)$ of integers such that there exist sets of arrows $\Lambda_{i}$ and $\Gamma_{i}, 0 \leq i \leq n$, of which only $\Lambda_{0}$ and $\Gamma_{0}$ can be empty, satisfying the following conditions:

(1) $i \neq j$ implies $\Lambda_{i} \cap \Lambda_{j}=\emptyset=\Gamma_{i} \cap \Gamma_{j}$,

(2) $\bigcup_{i=0}^{n} \Lambda_{i}$ is a complete set of arrows for $\operatorname{rad} A e$,

(3) $\bigcup_{i=0}^{n} \Gamma_{i}$ is a complete set of arrows for $\operatorname{rad} e A$,

(4) If $i \neq j$, or $i=0$, or $j=0$, then $a \in \Lambda_{i}$ and $b \in \Gamma_{j}$ implies $a b=0$,

(5) $N=n+\operatorname{card} \Lambda_{0}$.

Let $N_{e}=\max \{N \mid$ there exists $n$ such that $(N, n) \in \mathscr{N}\}$ and $n_{e}=\min \left\{n \mid\left(N_{e}, n\right)\right.$ $\in \mathscr{N}\}$.

Theorem 2.1 ([2]). Let $S$ be a noninjective simple A-module, and let e be a primitive idempotent of $A$ such that $S \cong e A / \operatorname{rad} e A$. The middle term of the almost split sequence starting at $S$ has exactly $N_{e}$ indecomposable direct summands. Furthermore, the number of indecomposable projective direct summands is equal to $N_{e}-n_{e}$.

Corollary $2.2([2])$. Let e be same as in Theorem 2.1 and $P=e A$. If $A$ is selfinjective, then the number of indecomposable direct summands of $\operatorname{rad} P / \operatorname{soc} P$ is equal to $n_{e}$.

2.2. A 2-cocycle induced by a cycle in the ordinary quiver. From now on, let $K$ be an algebraically closed field, $\Delta$ a quiver and $A:=K \Delta / R_{\Delta}^{n}(n \geq 2)$ a truncated quiver algebra such that any oriented cycle in $\Delta$ is zero in $A$. We assume that $\operatorname{dim} A>1$.

Since $A$ is a truncated quiver algebra, we can take a set $\mathbb{M}:=\left\{p_{i} \mid i=1, \ldots, t\right\}$ of paths in $\Delta$ such that $\left\{\overline{p_{i}} \mid i=1, \ldots, t\right\}$ is a basis of $\operatorname{soc}_{A^{e}} A$. Moreover, let $\left\{\overline{p_{1}}, \ldots, \overline{p_{t}}, \ldots, \overline{p_{d}}\right\}$ be a basis of $A$ by taking paths $p_{t+1}, \ldots, p_{d}$ in $\Delta$. We denote by $\left\{\bar{p}_{1}^{*}, \ldots,{\overline{p_{t}}}^{*}, \ldots,{\overline{p_{d}}}^{*}\right\}$ the dual basis in $D(A)$. We note that, by [3, Proposition 2.2.], the ordinary quiver $\Delta_{T_{0}(A)}$ is given by

- $\left(\Delta_{T_{0}(A)}\right)_{0}=\Delta_{0}$,

- $\left(\Delta_{T_{0}(A)}\right)_{1}=\Delta_{1} \cup\left\{y_{p_{1}}, \ldots, y_{p_{t}}\right\}$,

where, for each $i, y_{p_{i}}$ is an arrow from $t\left(p_{i}\right)$ to $s\left(p_{i}\right)$.

Next, under the notation of [5] and [6], we will define a 2-cocycle $\alpha$. For $n+$ $1 \leq s \leq 2 n-2$, let $\gamma=x_{1} x_{2} \cdots x_{s} \in \Delta_{s}^{c}$ be a cycle. Then it is easy to check that $\gamma$ is a basic cycle. We regard the subscripts $i$ of $x_{i}$ modulo $s(1 \leq i \leq s)$. Moreover, $\left(\left(A \otimes_{A^{e}} \boldsymbol{P}_{*}\right)_{s},\left(\tilde{d}_{*}\right)_{s}\right)$ is $\Delta_{s}^{c} / C_{s}$-graded and $\left\{v_{i}=x_{i+n} \cdots x_{i+s-1} \otimes_{K \Delta_{0}^{e}}\right.$ $\left.x_{i} x_{i+1} \cdots x_{i+n-1} \mid 1 \leq i \leq s\right\}$ is a basis of $\left(\left(A \otimes_{K \Delta_{0}^{e}} K \Delta_{n}\right)_{s}\right)_{\bar{\gamma}}$. We denote by $\left\{v_{i}^{*} \mid 1 \leq i \leq s\right\}$ the dual basis in $D\left(\left(\left(A \otimes_{K \Delta_{0}^{e}} K \Delta_{n}\right)_{s}\right)_{\bar{\gamma}}\right)$. Then we have the following complex

$$
\begin{aligned}
D\left(\left(\left(A \otimes_{K \Delta_{0}^{e}} K \Delta_{1}\right)_{s}\right)_{\bar{\gamma}}\right) \stackrel{0}{\longrightarrow} & D\left(\left(\left(A \otimes_{K \Delta_{0}^{e}} K \Delta_{n}\right)_{s}\right)_{\bar{\gamma}}\right) \\
& \stackrel{D\left(\left(\left(\tilde{d_{3}}\right)_{s}\right)_{\bar{\gamma}}\right)}{\longrightarrow} D\left(\left(\left(A \otimes_{K \Delta_{0}^{e}} K \Delta_{n+1}\right)_{s}\right) \bar{\gamma}\right),
\end{aligned}
$$


and we have the following isomorphism

$$
D\left(H H_{2, s, \bar{\gamma}}(A)\right) \cong \operatorname{Ker}\left(D\left(\left(\left(\tilde{d}_{3}\right)_{s}\right)_{\bar{\gamma}}\right)\right)=\left\langle v_{1}^{*}+\cdots+v_{s}^{*}\right\rangle .
$$

We denote the map $\Theta\left(v_{i}^{*}\right): A \times A \longrightarrow D(A)$ by $\alpha_{i}$ for $i=1,2, \ldots, s$. Then each $\alpha_{i}$ is the map as follows:

$$
\alpha_{i}(\bar{a}, \bar{b})= \begin{cases}{\overline{x_{i+m} \cdots x_{i+s-1}}}^{*} & \text { if } \bar{a}, \bar{b} \neq 0 \text { in } A, n \leq m<s \\ & \text { and } a b=x_{i} \cdots x_{i+m-1}, \\ {\overline{s\left(x_{i}\right)}}^{*} & \text { if } \bar{a}, \bar{b} \neq 0 \text { in } A \text { and } a b=x_{i} \cdots x_{i+s-1}, \\ 0 & \text { otherwise, }\end{cases}
$$

where $a, b$ are paths in $\Delta, m$ denotes the length of $a b$. Moreover, $\sum_{i=1}^{s} \alpha_{i}$ is a 2-cocycle and the cohomology class $\left[\sum_{i=1}^{s} \alpha_{i}\right]$ is a basis of $D\left(H H_{2, s}, \bar{\gamma}(A)\right)$. We fix a nonzero element $k(\neq 0) \in K$ and let $\alpha=k \sum_{i=1}^{s} \alpha_{i}$. Then we have the following proposition.

Proposition 2.3. The ordinary quiver of $T_{\alpha}(A)$ coincides with $\Delta_{T_{0}(A)}$.

Proof. We can prove this proposition by a similar way to [5, Theorem 4.3].

2.3. Elementary cycles and $\alpha$-revived cycles. Let $\alpha=k \sum_{i=1}^{s} \alpha_{i}$ be the 2cocycle defined in Section 2.2. We define an elementary cycle and its weight for $T_{\alpha}(A)$ based on [3, Definition 3.1]. Let $C$ be an oriented cycle in $\Delta_{T_{\alpha}(A)}$. We say that $C$ is elementary if $C=\delta_{2} y_{p_{i}} \delta_{1}$ for some paths $\delta_{1}$ and $\delta_{2}$ in $K \Delta$ and $p_{i} \in \mathbb{M}$ such that $\bar{p}_{i}^{*}\left(\overline{\delta_{1} \delta_{2}}\right) \neq 0$. Now let $C=a_{1} \cdots a_{j}$ be an oriented cycle in $\Delta_{T_{\alpha}(A)}$ where $a_{1}, \ldots, a_{j} \in \Delta_{1}$. We say that $C$ is $\alpha$-revived if there exist $a, b \in \Delta_{+}$such that $\bar{a}, \bar{b} \neq 0$ in $A, C=a_{1} \cdots a_{j}=a b$ and $\alpha(\bar{a}, \bar{b}) \neq 0$. Then, under the notation above, it is easy to see that $j=s, C=x_{i} \cdots x_{i+s-1}$ for some $i$ and $\alpha(\bar{a}, \bar{b})\left(1_{A}\right)=k$, where $k$ is the fixed element in the above. Moreover, we define a weight $w(C)$ of an elementary cycle $C=\delta_{2} y_{p_{i}} \delta_{1}$ by ${\overline{p_{i}}}^{*}\left(\overline{\delta_{1} \delta_{2}}\right)$, and we also define a weight $w(C)$ of an $\alpha$-revived cycle $C$ by $k$.

We say that a path $q$ is contained in a path $q^{\prime}$, if $q^{\prime}=\gamma_{1} q \gamma_{2}$, where $\gamma_{1}, \gamma_{2}$ are paths with $t\left(\gamma_{1}\right)=s(q)$ and $s\left(\gamma_{2}\right)=t(q)$.

Remark 2.1 (cf. [3, Remark 3.3]). If $0 \neq \bar{v} \in A$, then there are paths $\delta_{1}, \delta_{2}$ in $K \Delta$ and $p_{j} \in \mathbb{M}$ such that ${\overline{p_{j}}}^{*}\left(\overline{\delta_{1} v \delta_{2}}\right) \neq 0$, and in particular, any nonzero path in $A$ is contained in an elementary cycle.

Remark 2.2. If $C=a_{1} \cdots a_{m}$ with $a_{1}, \ldots, a_{m} \in\left(\Delta_{T_{\alpha}(A)}\right)_{1}$ is an elementary cycle, then $a_{2} a_{3} \cdots a_{m} a_{1}$ is also an elementary cycle.

Remark 2.3. If $C=a_{1} \cdots a_{j}$ with $a_{1}, \ldots, a_{j} \in \Delta_{1}$ is an $\alpha$-revived cycle, then $a_{2} a_{3} \cdots a_{j} a_{1}$ is also an $\alpha$-revived cycle.

Definition 1 (cf. [3, Definition 3.4]). Let $q$ be a path contained in an elementary cycle $C$ of length less than or equal to the length of $C$. The supplement of $q$ in $C$ is defined as follows:

$$
\begin{cases}\text { the trivial path } e_{s(q)} & \text { if } s(q)=t(q), \\ \text { the path formed by the remaining arrows of } C & \text { if } s(q) \neq t(q) .\end{cases}
$$


2.4. Example. We illustrate an example of the ordinary quiver of a Hochschild extension algebra and we give some examples of elementary cycles and $\alpha$-revived cycles.

Example 1. Let $\Delta$ be the following quiver:

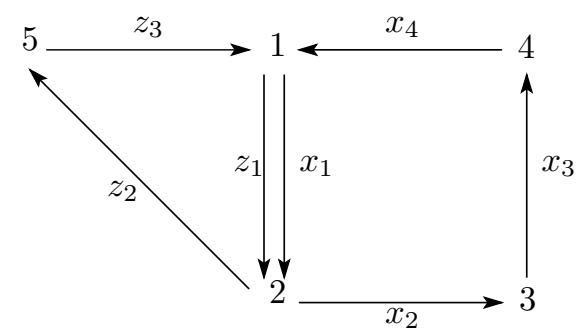

and $A=K \Delta / R_{\Delta}^{3}$. Let $\mathbb{M}$ be the set of paths of length 2. Then $\mathbb{M}$ forms a $K$-basis of $\operatorname{soc}_{A^{e}} A$ of $\operatorname{dim}_{K} \operatorname{soc}_{A^{e}} A=11$. We put $\gamma=x_{1} x_{2} x_{3} x_{4}$. For each $i(1 \leq i \leq 4)$, $\alpha_{i}: A \times A \longrightarrow D(A)$ corresponding to $\gamma$ is the map as follows:

$$
\alpha_{i}(\bar{a}, \bar{b})= \begin{cases}{\overline{x_{i+3}}}^{*} & \text { if } \bar{a}, \bar{b} \neq 0 \text { in } A \text { and } a b=x_{i} x_{i+1} x_{i+2}, \\ \bar{e}_{i+4} & \text { if } \bar{a}, \bar{b} \neq 0 \text { in } A \text { and } a b=x_{i} x_{i+1} x_{i+2} x_{i+3} \\ 0 & \text { otherwise. }\end{cases}
$$

Let $k(\neq 0) \in K$ and $\alpha=k \sum_{i=1}^{4} \alpha_{i}$. Then, the ordinary quiver of $T_{\alpha}(A)$ coincides with $\Delta_{T_{0}(A)}$, and $\Delta_{T_{0}(A)}$ is the following quiver:

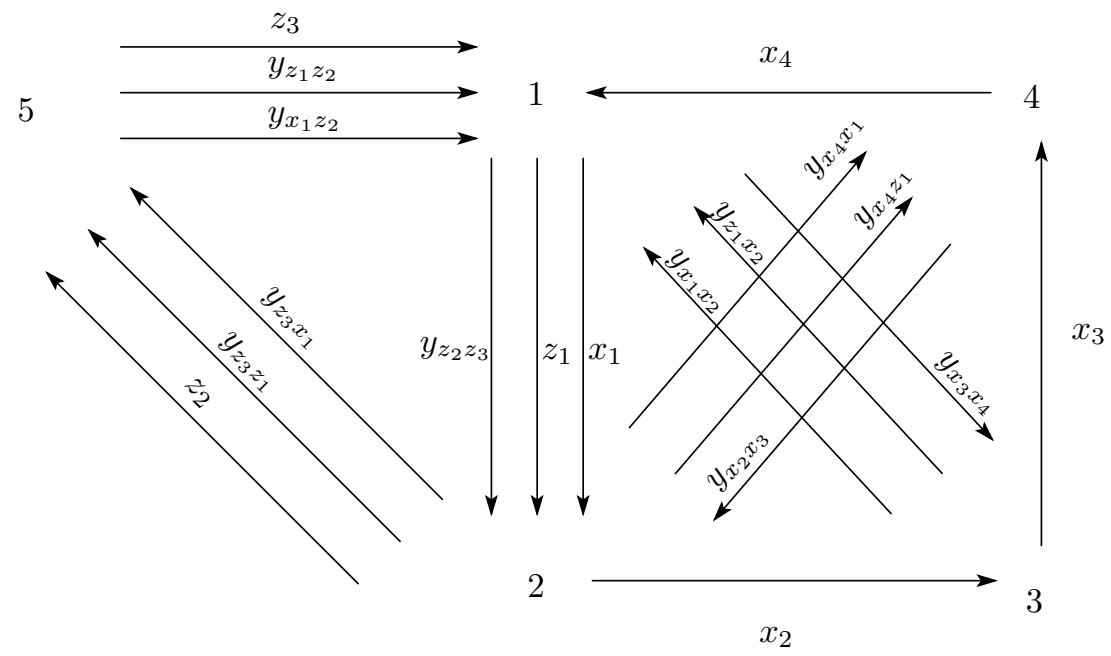

The elementary cycles with origin 1 are

$$
\begin{array}{lcccc}
y_{z_{2} z_{3}} z_{2} z_{3}, & z_{1} y_{z_{3} z_{1}} z_{3}, & x_{1} y_{z_{3} x_{1}} z_{3}, & z_{1} z_{2} y_{z_{1} z_{2}}, & x_{1} z_{2} y_{x_{1} z_{2}}, \\
x_{1} x_{2} y_{x_{1} x_{2}}, & z_{1} x_{2} y_{z_{1} x_{2}}, & x_{1} y_{x_{4} x_{1}} x_{4}, & z_{1} y_{x_{4} z_{1}} x_{4}, & y_{x_{3} x_{4}} x_{3} x_{4}
\end{array}
$$

Moreover, there is only one $\alpha$-revived cycle with origin 1 , which is $\gamma=x_{1} x_{2} x_{3} x_{4}$.

For the elementary cycle $y_{z_{2} z_{3}} z_{2} z_{3}$, the supplement of $y_{z_{2} z_{3}} z_{2}$ in $y_{z_{2} z_{3}} z_{2} z_{3}$ is $z_{3}$ and the supplement of $z_{2}$ in $y_{z_{2} z_{3}} z_{2} z_{3}$ is $z_{3} y_{z_{2} z_{3}}$. 


\section{Nonzero ORIENTEd CYCLES IN $T_{\alpha}(A)$}

Let $T_{\alpha}(A)$ be a Hochschild extension algebra same as in Section 2. We note that the ordinary quiver $\Delta_{T_{\alpha}(A)}$ coincides with $\Delta_{T_{0}(A)}$ which is given by

- $\left(\Delta_{T_{0}(A)}\right)_{0}=\Delta_{0}$,

- $\left(\Delta_{T_{0}(A)}\right)_{1}=\Delta_{1} \cup\left\{y_{p_{1}}, \ldots, y_{p_{t}}\right\}$,

where, for each $i, y_{p_{i}}$ is an arrow from $t\left(p_{i}\right)$ to $s\left(p_{i}\right)$.

In this section, we give a characterization of nonzero oriented cycles in $T_{\alpha}(A)$. We will show that a nonzero oriented cycle is either an elementary cycle or an $\alpha$-revived cycle.

Consider the homomorphism of $K$-algebras $\Phi: K \Delta_{T_{\alpha}(A)} \longrightarrow T_{\alpha}(A)$ defined on the trivial paths and the arrows as follows:

$$
\begin{aligned}
\Phi\left(e_{i}\right) & =\left(\overline{e_{i}}, 0\right) \text { for } i=1, \ldots, n, \\
\Phi(a) & =(\bar{a}, 0) \text { for } a \in \Delta_{1}, \\
\Phi\left(y_{p_{i}}\right) & =\left(0,{\overline{p_{i}}}^{*}\right) \text { for } p_{i} \in \mathbb{M} .
\end{aligned}
$$

Then $\Phi$ is surjective. Let $\pi_{1}$ and $\pi_{2}$ are the projections induced by the decomposition $T_{\alpha}(A)=A \oplus D(A)$, and we define

$$
\varphi_{1}:=\pi_{1} \Phi: K \Delta_{T_{\alpha}(A)} \longrightarrow A \quad \text { and } \quad \varphi_{2}:=\pi_{2} \Phi: K \Delta_{T_{\alpha}(A)} \longrightarrow D(A) .
$$

In this paper, $Y$ denotes the ideal generated by the elements $y_{p_{i}}(i=1, \ldots, t)$ in $K \Delta_{T_{\alpha}(A)}$.

We show the following lemma.

Lemma 3.1. Suppose $z$ is a path in $K \Delta_{T_{\alpha}(A)}$. Then the following hold

(1) $\Phi(z)=0$ if $z$ contains two or more arrows $y_{p_{i}}(i=1, \ldots, t)$.

(2) If $v=v_{1}+v_{2}$ with a path $v_{1} \in K \Delta$ and a path $v_{2} \in Y$, then

$$
\Phi(v)= \begin{cases}\left(\varphi_{1}\left(v_{1}\right), \varphi_{2}\left(v_{2}\right)\right) & \text { if length } v_{1}<n, \\ \left(0, \varphi_{2}\left(v_{1}+v_{2}\right)\right) & \text { if } n \leq \text { length } v_{1} \leq s, \\ \left(0, \varphi_{2}\left(v_{2}\right)\right) & \text { if } s<\text { length } v_{1} .\end{cases}
$$

(3) $\varphi_{2}(z) \neq 0$ implies that $z \in Y$ or $n \leq$ length $z \leq s$.

(4) Let $C$ be an elementary cycle or an $\alpha$-revived cycle with origin e. Then, for any path $u(\neq e)$ in $K \Delta, \varphi_{2}(C)(\bar{e})=w(C)$ and $\varphi_{2}(C)(\bar{u})=0$.

(5) If $u$ is a path from $i$ to $j$ in $K \Delta, v$ is a path from $j$ to $i$ in $K \Delta_{T_{\alpha}(A)}$ and both $u v$ and $v u$ are not $\alpha$-revived cycles, then $\varphi_{2}(v)(\bar{u})=\varphi_{2}(v u)\left(\overline{e_{j}}\right)=\varphi_{2}(u v)\left(\overline{e_{i}}\right)$.

(6) If $u$ is a path from $i$ to $j$ in $K \Delta, v$ is a path from $j$ to $i$ in $K \Delta_{T_{\alpha}(A)}$ and both $u v$ and $v u$ are not $\alpha$-revived cycles, then $v u \in \operatorname{Ker} \Phi$ if and only if uv $\in \operatorname{Ker} \Phi$.

(7) Let $u$ is a path in $K \Delta$ and $z$ a path in $Y$. Then $\varphi_{2}(z)(\bar{u}) \neq 0$ implies that $u$ is a supplement of $z$.

Proof. We get (1) using that $D(A)^{2}=0$ in $T_{\alpha}(A)$. (2) and (3) follow directly from the definitions.

(4) If $C$ is an elementary cycle, then (4) follows from the definitions together with the hypothesis over $A$. If $C$ is an $\alpha$-revived cycle, $C=e x_{i} x_{i+1} \cdots x_{i+s-1}$ for 
some $i \in\{1, \ldots s\}$. So we have

$$
\begin{aligned}
\Phi(C) & =\left(\overline{x_{i} x_{i+1} \cdots x_{i+n-1}}, \alpha\left(\overline{x_{i}}, \overline{x_{i+1} \cdots x_{i+n-1}}\right)\right)\left(\overline{x_{i+n}}, 0\right) \cdots\left(\overline{x_{i+s-1}}, 0\right) \\
& =\left(0, \alpha\left(\overline{x_{i}}, \overline{x_{i+1} \cdots x_{i+n-1}}\right)\right)\left(\overline{x_{i+n}}, 0\right) \cdots\left(\overline{x_{i+s-1}}, 0\right) \\
& =\left(0, \alpha\left(\overline{x_{i}}, \overline{x_{i+1} \cdots x_{i+n-1}}\right) \cdot\left(\overline{x_{i+n} \cdots x_{i+s-1}}\right)\right)
\end{aligned}
$$

and

$$
\begin{aligned}
\varphi_{2}(C)(\bar{e}) & =\left(\alpha\left(\overline{x_{i}}, \overline{x_{i+1} \cdots x_{i+n-1}}\right) \cdot\left(\overline{x_{i+n} \cdots x_{i+s-1}}\right)\right)(\bar{e}) \\
& =w(C) \overline{x_{i+n} \cdots x_{i+s-1}} *\left(\overline{e x_{i+n} \cdots x_{i+s-1}}\right) \\
& =w(C) .
\end{aligned}
$$

It is clear that $\varphi_{2}(C)(\bar{u})=0$ for any path $u$.

(5) First, we consider the case $\bar{u}=0$ in $A$. We have $\varphi_{2}(v)(\bar{u})=0$. On the other hand, since

$$
\Phi(u)= \begin{cases}(\bar{u}, 0) & \text { if length } u<n, \\ \left(0, \varphi_{2}(u)\right) & \text { if } n \leq \text { length } u \leq q, \\ 0 & \text { otherwise }\end{cases}
$$

we have

$$
\begin{aligned}
\varphi_{2}(v u)\left(\overline{e_{j}}\right) & =\pi_{2}(\Phi(v) \Phi(u))\left(\overline{e_{j}}\right) \\
& = \begin{cases}\pi_{2}(\Phi(v)(\bar{u}, 0))\left(\overline{e_{j}}\right) & \text { if length } u<n, \\
\pi_{2}\left(\Phi(v)\left(0, \varphi_{2}(u)\right)\right)\left(\overline{e_{j}}\right) & \text { if } n \leq \text { length } u \leq s, \\
0 & \text { otherwise. }\end{cases}
\end{aligned}
$$

If length $u \leq n$ then $\pi_{2}(\Phi(v)(\bar{u}, 0))\left(\overline{e_{j}}\right)=0$ by $\bar{u}=0$ in $A$. So we consider the case that $n \leq$ length $u \leq s$. We put $m=$ length $u$. Then $u=a_{1} a_{2} \cdots a_{m}$ for some arrows $a_{1}, a_{2}, \ldots a_{m} \in \Delta$, and we have

$$
\begin{aligned}
& \varphi_{2}(u) \\
& =\pi_{2}\left(\left(\overline{a_{1}}, 0\right)\left(\overline{a_{2}}, 0\right) \cdots\left(\overline{a_{n}}, 0\right)\left(\overline{a_{n+1}}, 0\right) \cdots\left(\overline{a_{m}}, 0\right)\right) \\
& =\pi_{2}\left(\left(\overline{a_{1} a_{2} \cdots a_{n}}, \alpha\left(\overline{a_{1} a_{2} \cdots a_{n-1} a_{n}}\right)\right)\left(\overline{a_{n+1}}, 0\right) \cdots\left(\overline{a_{m}}, 0\right)\right) \\
& =\left\{\begin{array}{cc}
\pi_{2}\left(\left(0, \overline{x_{i+n} \cdots x_{i+s-1}}\right)\left(\overline{a_{n+1}}, 0\right) \cdots\left(\overline{a_{m}}, 0\right)\right) & \text { if } a_{1} \cdots a_{n}=x_{i} \cdots x_{i+n-1} \\
\text { for some } i \in\{1, \ldots, s\}, & \text { otherwise }
\end{array}\right. \\
& =\left\{\begin{array}{cc}
\pi_{2}\left((0,0)\left(\overline{a_{n+1}}, 0\right) \cdots\left(\overline{a_{m}}, 0\right)\right) & \text { for some } i \in\{1, \ldots, s\}, \\
\pi_{2}\left(0, \overline{x_{i+n} \cdots x_{i+s-1}} \cdot \overline{a_{n+1} \cdots a_{m}}\right) & \text { if } a_{1} \cdots a_{n}=x_{i} \cdots x_{i+n-1}
\end{array}\right. \\
& =\left\{\begin{array}{cc}
\overline{x_{i+n} \cdots x_{i+s-1}} \cdot \overline{a_{n+1} \cdots a_{m}} & \text { if } a_{1} \cdots \\
\text { for some } i \in\{1, \ldots, s\}, \\
0 & \text { otherwise. }
\end{array}\right.
\end{aligned}
$$


On the other hand, $\Phi(v)=(\bar{v}, 0)$ or $\Phi(v)=(0, f)$ for some $f \in D(A)$. So we have

$$
\begin{aligned}
& \pi_{2}\left(\Phi(v)\left(0, \varphi_{2}(u)\right)\right)\left(\overline{e_{j}}\right) \\
& = \begin{cases}\pi_{2}\left((\bar{v}, 0)\left(0, \varphi_{2}(u)\right)\right)\left(\overline{e_{j}}\right) & \text { if } \Phi(v)=(\bar{v}, 0), \\
0 & \text { otherwise }\end{cases} \\
& = \begin{cases}\pi_{2}\left(0, \bar{v} \cdot \overline{x_{i+n} \cdots x_{i+s-1}} \cdot \overline{a_{n+1} \cdots a_{m}}\right)\left(\overline{e_{j}}\right) & \text { if } \Phi(v)=(\bar{v}, 0) \text { and } \\
a_{1} \cdots a_{n}=x_{i} \cdots x_{i+n-1} & \text { for some } i \in\{1, \ldots, s\}, \\
0 & \text { otherwise }\end{cases} \\
& = \begin{cases}\overline{x_{i+n} \cdots x_{i+s-1}} *\left(\overline{a_{n+1} \cdots a_{m} v}\right) & \text { if } \Phi(v)=(\bar{v}, 0) \text { and } \\
a_{1} \cdots a_{n}=x_{i} \cdots x_{i+n-1} & \text { for some } i \in\{1, \ldots, s\}, \\
0 & \text { otherwise. }\end{cases}
\end{aligned}
$$

If $\overline{x_{i+n} \cdots x_{i+s-1}} *\left(\overline{a_{n+1} \cdots a_{m} v}\right) \neq 0$, then $u v=a_{1} \cdots a_{m} v=x_{i} \cdots x_{i+s-1}$. This contradicts that $u v$ is not an $\alpha$-revived cycle.

Next we consider the case $\bar{u} \neq 0$ in $A$. Since $v$ is a path, by Lemma 3.1 (2), $\Phi(v)=(\bar{v}, 0)$ or $\Phi(v)=(0, f)$ for some $f \in D(A)$. Since $v u$ is not an $\alpha$-revived cycle, if $\Phi(v)=(\bar{v}, 0)$, then we have $\alpha(\bar{v}, \bar{u})=0$ and

$$
\varphi_{2}(v u)\left(\overline{e_{j}}\right)=\pi_{2}((\bar{v}, 0)(\bar{u}, 0))\left(\overline{e_{j}}\right)=\pi_{2}(\overline{v u}, \alpha(\bar{v}, \bar{u}))\left(\overline{e_{j}}\right)=\pi_{2}(\overline{v u}, 0)\left(\overline{e_{j}}\right)=0 .
$$

On the other hand, $\varphi_{2}(v)(\bar{u})=\pi_{2}(\bar{v}, 0)(\bar{u})=0$. If $\Phi(v)=(0, f)$, then we have

$$
\varphi_{2}(v u)\left(\overline{e_{j}}\right)=\pi_{2}((0, f)(\bar{u}, 0))\left(\overline{e_{j}}\right)=(f \bar{u})\left(\overline{e_{j}}\right)=\pi_{2}(0, f)(\bar{u})=\varphi_{2}(v)(\bar{u}) .
$$

Similarly, $\varphi_{2}(v)(\bar{u})=\varphi_{2}(v u)\left(\overline{e_{j}}\right)$.

(6) Since $v u$ and $u v$ are not $\alpha$-revived cycles, it suffices to consider the case $v u, u v \in Y$. By the assumption that any oriented cycle in $K \Delta$ is zero in $A$, for any path $x$ in $\Delta$,

$$
\varphi_{2}(v u)(\bar{x})= \begin{cases}\varphi_{2}(v u)\left(\overline{e_{j}}\right) & \text { if } \bar{x}=\overline{e_{j}} \\ 0 & \text { otherwise }\end{cases}
$$

and

$$
\varphi_{2}(u v)(\bar{x})= \begin{cases}\varphi_{2}(u v)\left(\overline{e_{i}}\right) & \text { if } \bar{x}=\overline{e_{i}} \\ 0 & \text { otherwise }\end{cases}
$$

If $v u \in \operatorname{Ker} \Phi$, by (5), then $\varphi_{2}(u v)\left(\overline{e_{i}}\right)=\varphi_{2}(v u)\left(\overline{e_{j}}\right)=0$. So we have $\varphi_{2}(u v)=0$, and $v u \in \operatorname{Ker} \Phi$ implies that $u v \in \operatorname{Ker} \Phi$. The converse holds, similarly.

(7) By (1), $z=\delta_{2} y_{p_{i}} \delta_{1}$ with paths $\delta_{1}, \delta_{2}$ in $K \Delta$ and $i \in\{1, \ldots, t\}$. Then $\bar{p}_{i}^{*}\left(\overline{\delta_{1} u \delta_{2}}\right)=\varphi(z)(\bar{u}) \neq 0$. Therefore $u$ is a supplement of $z$ in the elementary cycle $\delta_{2} y_{p_{i}} \delta_{1} u$.

Theorem 3.2. Let $C$ be an oriented cycle in $K \Delta_{T_{\alpha}(A)}$. Then the following conditions are equivalent:

(i) $C$ is an elementary cycle or $\alpha$-revived cycle.

(ii) $C$ is nonzero in $T_{\alpha}(A)$. 
Proof. By Lemma 3.1 (4), (i) implies (ii). We will show that (ii) implies (i). Let $C_{1}$ be a cycle in $K \Delta$, that is, $C_{1}=z_{1} \cdots z_{m}$ for some $m \geq n$ and $z_{1}, \ldots, z_{m} \in \Delta_{1}$. We assume that $\varphi_{2}\left(C_{1}\right) \neq 0$ and we will show that $C_{1}$ is an $\alpha$-revived cycle. By Lemma $3.1(2), \Phi\left(C_{1}\right)=\left(0, \varphi_{2}\left(C_{1}\right)\right)$. By Lemma $3.1(2)$, we have $n \leq m \leq s$ and

$$
\begin{aligned}
0 & \neq \varphi_{2}\left(C_{1}\right) \\
& =\pi_{2}\left(\left(\overline{z_{1}}, 0\right) \cdots\left(\overline{z_{m}}, 0\right)\right) \\
& =\pi_{2}\left(\left(0, \alpha\left(\overline{z_{1} \cdots z_{n-1}}, \overline{z_{n}}\right)\right)\left(\overline{z_{n+1}}, 0\right) \cdots\left(\overline{z_{m}}, 0\right)\right) \\
& =\alpha\left(\overline{z_{1} \cdots z_{n-1}}, \overline{z_{n}}\right) \cdot \overline{z_{n+1} \cdots z_{m}} .
\end{aligned}
$$

So $\alpha\left(\overline{z_{1} \cdots z_{n-1}}, \overline{z_{n}}\right) \neq 0$, we have $z_{1} \cdots z_{n}$ coincides with $x_{i} \cdots x_{i+n-1}$ and $\alpha\left(\overline{z_{1} \cdots z_{n-1}}, \overline{z_{n}}\right)=$ $\left(\overline{x_{i+n} \cdots x_{i+s-1}}\right)^{*}$ for some $i \in\{1, \ldots, s\}$. Since

$$
0 \neq \alpha\left(\overline{z_{1} \cdots z_{n-1}}, \overline{z_{n}}\right) \cdot \overline{z_{n+1} \cdots z_{m}}=\overline{x_{i+n} \cdots x_{i+s-1}} * \overline{z_{n+1} \cdots z_{n}},
$$

there exists a path $\delta$ in $\Delta$ such that

$$
0 \neq\left(\overline{x_{i+n} \cdots x_{i+s-1}} * \overline{z_{n+1} \cdots z_{n}}\right)(\bar{\delta})={\overline{x_{i+n} \cdots x_{i+s-1}}}^{*}\left(\overline{z_{n+1} \cdots z_{n}} \bar{\delta}\right) .
$$

Then $z_{n+1} \cdots z_{m} \delta=x_{i+n} \cdots x_{i+s-1}$ and $z_{1} \cdots z_{m} \delta$ coincides with an $\alpha$-revived cycle $x_{i} \cdots x_{i+n-1} x_{i+n} \cdots x_{i+s-1}$. Therefore, by that $C_{1}=z_{1} \cdots z_{m}$ is a cycle, $\delta$ is a trivial path and $C_{1}$ is an $\alpha$-revived cycle.

Next, let $C_{2}$ be a cycle from $j$ to $j$ in $Y$. We assume that $\Phi\left(C_{2}\right) \neq 0$. We have $\Phi\left(C_{2}\right)=\left(0, \varphi_{2}\left(C_{2}\right)\right)$, by Lemma $3.1(2)$. By Lemma 3.1 (1), suppose now that $C_{2}$ contains exactly one arrow $y_{p_{i}}$. Then $C_{2}=\delta_{2} y_{p_{i}} \delta_{1}$ with $\delta_{1}, \delta_{2}$ paths in $K \Delta$. Since $\varphi_{2}\left(C_{2}\right) \neq 0$, there exists a path $u$ in $K \Delta$ such that $\varphi_{2}\left(C_{2}\right)(\bar{u}) \neq 0$. Then $u$ is a path from $j$ to $j$ and $\bar{u} \neq 0$. So, by the hypothesis on $A, u=e_{j}$ and thus $C_{2}$ is an elementary cycle.

Therefore, any nonzero oriented cycle is elementary or $\alpha$-revived.

Proposition 3.3. For each $j \in\left(\Delta_{T_{\alpha}(A)}\right)_{0}$, let $I_{j}$ be the ideal in $K \Delta_{T_{\alpha}(A)}$ generated by

(i) oriented cycles in $K \Delta_{T_{\alpha}(A)}$ from $j$ to $j$ which are neither elementary nor $\alpha$-revived,

(ii) elements $w\left(C^{\prime}\right) C-w(C) C^{\prime} \in K \Delta_{T_{\alpha}(A)}$, where $C, C^{\prime}$ are elementary or $\alpha$-revived cycles with origin $j$.

Then $\operatorname{Ker} \Phi \cap e_{j} K \Delta_{T_{\alpha}(A)} e_{j}$ generates $I_{j}$.

Proof. By the proof of Theorem 3.2, oriented cycles from $j$ to $j$ which are neither elementary nor $\alpha$-revived are in $\operatorname{Ker} \Phi \cap e_{j} K \Delta_{T_{\alpha}(A)} e_{j}$. Let now $z=w\left(C^{\prime}\right) C-$ $w(C) C^{\prime}$ be an element as defined in (ii). Then $\Phi(z)=\left(0, \varphi_{2}(z)\right)$, and $\varphi_{2}(z)=$ $w\left(C^{\prime}\right) \varphi_{2}(C)-w(C) \varphi_{2}\left(C^{\prime}\right)$. By Lemma $3.1(4)$, for any path $u$ in $K \Delta$,

$$
\begin{aligned}
\varphi_{2}(z)(\bar{u}) & =\left(w\left(C^{\prime}\right) \varphi_{2}(C)\right)(\bar{u})-\left(w(C) \varphi_{2}\left(C^{\prime}\right)\right)(\bar{u}) \\
& = \begin{cases}w\left(C^{\prime}\right) w(C)-w(C) w\left(C^{\prime}\right) & \text { if } u=e_{j}, \\
0 & \text { otherwise }\end{cases} \\
& =0 .
\end{aligned}
$$

Hence, $z \in \operatorname{Ker} \Phi \cap e_{j} K \Delta_{T_{\alpha}(A)} e_{j}$.

Thus $I_{j}$ is contained in the ideal generated by $\operatorname{Ker} \Phi \cap e_{j} K \Delta_{T_{\alpha}(A)} e_{j}$. The proof of the other inclusion is similar to the proof of [3, Proposition 3.6]. 


\section{An application of a theorem of Brenner}

In this section, we give the number of indecomposable direct summands of the middle term of almost split sequence for $T_{\alpha}(A)$. We define a relation on the set of nonzero oriented cycles with same origin in $\Delta_{T_{\alpha}(A)}$. We will show that the above number is equal to the cardinality of the equivalence classes.

The following proposition is a generalization of Remark 2.1.

Proposition 4.1. Any nonzero path in $T_{\alpha}(A)$ is contained in a nonzero oriented cycle.

Proof. Let $w$ be a nonzero path in $T_{\alpha}(A)$. Then $w$ is in $K \Delta$ or in $Y$. First, we suppose that $w \in K \Delta$. Since $\Phi(w) \neq 0$, we have length $w \leq s$ by Lemma 3.1 (2). If $n \leq$ length $w \leq s$, by the construction of $\alpha$ and the same calculation as in the proof of Lemma $3.1(5), w$ is contained in an $\alpha$-revived cycle. If length $w<n$, then $w \neq 0$ in $A$ and $w$ is in an elementary cycle by Remark 2.1.

Next, we suppose that $w \in Y$. We have $\varphi_{2}(w) \neq 0$. Hence $\varphi_{2}(w)(\bar{u}) \neq 0$ for some path $u$ in $K \Delta$. By Lemma $3.1(7), u$ is a supplement of $w$, so $w$ is contained in an elementary cycle $w u$.

Definition 2. For each $h \in\left(\Delta_{T_{\alpha}(A)}\right)_{0}$, let us denote by $\mathscr{C}_{h}$ the set of all oriented cycles $C$ such that $C \neq 0$ in $T_{\alpha}(A)$ and $s(C)=t(C)=h$. Let $C, C^{\prime}$ be in $\mathscr{C}_{h}$. If there exists an arrow $a$ belonging to $C$ and $C^{\prime}$ with $s(a)=h$ or $t(a)=h$, then we write $C \mathscr{R} C^{\prime}$.

Definition 3. For each $h \in\left(\Delta_{T_{\alpha}(A)}\right)_{0}$, let $\mathscr{A}_{h}=\left\{a \in\left(\Delta_{T_{\alpha}(A)}\right)_{1} \mid t(a)=h\right\}$. For $a, a^{\prime} \in \mathscr{A}_{h}$, if there exists an arrow $b \in\left(\Delta_{T_{\alpha}(A)}\right)_{1}$ such that $a b \neq 0$ and $a^{\prime} b \neq 0$ in $T_{\alpha}(A)$ then we write $a \mathscr{R}^{\prime} a^{\prime}$.

We note that, for any path $a \in \mathscr{A}_{h}, a \mathscr{R}^{\prime} a$ holds by Proposition 4.1.

From now on, we denote by " $\equiv "$ and " $\approx$ " the equivalence relations generated by $\mathscr{R}$ in $\mathscr{C}_{h}$ and by $\mathscr{R}^{\prime}$ in $\mathscr{A}_{h}$, respectively.

We next want to give the precise connection between these equivalence relations. For this purpose, the following results will be useful.

Proposition 4.2. $\operatorname{card}\left(\mathscr{C}_{h} / \equiv\right)=\operatorname{card}\left(\mathscr{A}_{h} / \approx\right)$.

Proof. For $C \in \mathscr{C}_{h}$, we denote by $a_{C}$ the last arrow of $C$, that is, $C=\delta a h$ for some path $\delta$. Consider the map $u: \mathscr{C}_{h} / \equiv \longrightarrow \mathscr{A}_{h} / \approx$ defined by $u(\bar{C})=\overline{a_{C}}$ for $C \in \mathscr{C}_{h}$. We will show that $u$ is well-defined. It suffices to show that if $C_{1} \mathscr{R} C_{2}$ then $a_{C_{1}} \mathscr{R}^{\prime} a_{C_{2}}$. We assume $C_{1} \mathscr{R} C_{2}$. Then $a_{C_{1}}=a_{C_{2}}$ or $C_{i}=b \delta_{i} a_{C_{i}}$ for some arrow $b$ and some path $\delta_{i}(i=1,2)$. If $a_{C_{1}}=a_{C_{2}}$ then $a_{C_{1}} \mathscr{R}^{\prime} a_{C_{2}}$, clearly. If $C_{i}=b \delta_{i} a_{C_{i}}$, by Theorem 3.2 , then $C_{i}$ is an elementary cycle or an $\alpha$-revived cycle $(\mathrm{i}=1,2)$. Thus, by Remark 2.2 and Remark 2.3, $a_{C_{i}} \delta_{i} b \neq 0$ and both $a_{1} b$ and $a_{2} b$ are not zero. So we have $a_{C_{1}} \mathscr{R}^{\prime} a_{C_{2}}$. And $u$ is well-defined. On the other hand, for $a \in \mathscr{A}_{h}$, by Proposition 4.1, there is a cycle $\delta_{1} a h \delta_{2}(\neq 0)$ for some path $\delta_{1}$ and $\delta_{2}$. By Remark 2.2 and Remark 2.3, $\delta_{2} \delta_{1} a h \neq 0$. We denote $C_{a}:=\delta_{2} \delta_{2} a h$. Then $\overline{C_{a}}$ does not depend on the choice of $\delta_{1}$ and $\delta_{2}$, that is, $\overline{C_{a}}$ is uniquely determined. We define the map $v: \mathscr{A}_{h} / \approx \longrightarrow \mathscr{C}_{h} / \equiv$ by $v(\bar{a})=\overline{C_{a}}$. We will show that $v$ is well-defined. If $a_{1} \mathscr{R}^{\prime} a_{2}$ then there exists a path $b$ such that $a_{1} b \neq 0$ and $a_{2} b \neq 0$. By Proposition 4.1, there is a cycle $\gamma_{i} a_{i} b \gamma_{i}^{\prime}$ for some path $\gamma_{i}$ and $\gamma_{i}^{\prime}(i=1,2)$. Thus we have

$$
C_{a_{1}} \mathscr{R}\left(b \gamma_{1}^{\prime} \gamma_{1} a_{1}\right) \mathscr{R}\left(b \gamma_{2}^{\prime} \gamma_{2} a_{2}\right) \mathscr{R} C_{a_{2}}
$$


So $C_{a_{1}} \equiv C_{a_{2}}$. It is easy to see that $u$ and $v$ are mutually inverse maps.

We have the following theorem, which is similar to [3, Proposition 4.9]:

Proposition 4.3. Let $h$ be a vertex in $\Delta_{T_{\alpha}(A)}$, and let $e_{h}$ be the idempotent element corresponding to $h$. Then we have $N_{e_{h}}=n_{e_{h}}=\operatorname{card}\left(\mathscr{C}_{h} / \equiv\right)$.

Proof. The proof is similar to [3, Proposition 4.9]. Let $\Lambda_{1}, \ldots, \Lambda_{t}$ be the equivalence classes of $\mathscr{A}_{h}$ by $\approx$. We put $\Gamma_{i}=\left\{b\right.$ : arrow $\mid \exists a \in \Lambda_{i}$ s.t. $\left.a b \neq 0\right\}$ for each $i(1 \leq$ $i \leq t$ ), and we set $\Lambda_{0}=\Gamma_{0}=\emptyset$. We note that $\Gamma_{i} \neq \emptyset$ for each $i=1, \ldots, t$. By construction, the pair $(t, t)$ is in $\mathscr{N}$. We shall prove that if $(N, n) \in \mathscr{N}$, then $N-n \geq t$. In fact, let $\Lambda_{i}^{\prime}$ and $\Gamma_{i}^{\prime}$ be sets of arrows satisfying Brenner's condition, for $0 \leq i \leq n$. Using the above conditions, we conclude that

(1) $\Gamma_{i}^{\prime}=\left\{b \mid\right.$ there exists $a \in \Lambda_{i}^{\prime}$ with $\left.a b \neq 0\right\}$ for $i=1, \ldots, n$,

(2) $\Gamma_{0}^{\prime}=\Lambda_{0}^{\prime}=\emptyset$.

It follows from (2) that $N=n$. On the other hand, it is easy to see that if $a \approx a^{\prime}$ then there exists $j$ such that $a, a^{\prime} \in \Lambda_{j}^{\prime}$. Then, for $1 \leq i \leq t$, there exists $j$ such that $\Lambda_{i} \subset \Lambda_{j}^{\prime}$, which implies $n \leq t$. Therefore, $N_{e_{h}}=n_{e_{h}}=\operatorname{card}\left(\mathscr{A}_{h} / \approx\right)=\operatorname{card}\left(\mathscr{C}_{h} / \equiv\right)$ by Proposition 4.2.

The following theorems are partial generalizations of [3].

Theorem 4.4. Let $S_{h}$ be the simple $T_{\alpha}(A)$-module corresponding to the vertex $h$. Then the number of indecomposable direct summands of the middle term of almost split sequence

$$
0 \longrightarrow S_{h} \longrightarrow E \longrightarrow \tau^{-1} S_{h} \longrightarrow 0
$$

is equal to the number of equivalence classes in $\mathscr{C}_{h}$. Furthermore, the number of indecomposable projective summands of $E$ is equal to zero.

Theorem 4.5. Let $P_{h}$ be the indecomposable projective $T_{\alpha}(A)$-module corresponding to the vertex $h$. Then the number of indecomposable direct summands of $\operatorname{rad} P_{h} / \operatorname{soc} P_{h}$ is equal to the number of equivalence classes in $\mathscr{C}_{h}$.

Corollary 4.6. Let $n \geq 3$ and $h \in \Delta_{0}$ be neither sink nor source in $\Delta$. Then we have $\operatorname{card}\left(\mathscr{C}_{h} / \equiv\right)=1$.

Proof. By Proposition 4.2 , we have $\operatorname{card}\left(\mathscr{C}_{h} / \equiv\right)=\operatorname{card}\left(\mathscr{A}_{h} / \approx\right)$. So we show $\operatorname{card}\left(\mathscr{A}_{h} / \approx\right)=1$. Let $\mathscr{A}_{h}=\left\{x_{1}, x_{2}, \ldots, x_{m}, y_{1}, y_{2}, \ldots, y_{l}\right\}$, where $x_{1}, \ldots, x_{m} \in \Delta_{0}$ and $y_{1}, \ldots, y_{l} \in\left(\Delta_{T_{\alpha}(A)}\right)_{0} \backslash \Delta_{0}$. Now $h$ is not sink in $\Delta$, so there exists an arrow $w \in \Delta_{1}$ such that $s(w)=h$. By the assumption that $A=K \Delta / R_{\Delta}^{n}$ and $n \geq 3$, we have $x_{i} w \neq 0$ in $A$ for any $1 \leq i \leq m$. So $x_{i} w \neq 0$ in $T_{\alpha}(A)$ and

$$
x_{1} \approx x_{2} \approx \cdots \approx x_{m}
$$

in $\mathscr{A}_{h}$.

Moreover, for any $j \in\{1, \ldots, l\}$, by Proposition $4.1, y_{j}$ is contained in the $\alpha$ revived cycle. Then we have $y_{j} v \neq 0$ in $T_{\alpha}(A)$ for some $v \in \Delta_{1}$. And, by the same reason as above, $x_{1} v \neq 0$ in $T_{\alpha}(A)$. So we have $x_{1} \approx y_{j}$ in $\mathscr{A}_{h}$. Hence we have $\operatorname{card}\left(\mathscr{C}_{h} / \equiv\right)=1$.

Example 2. Let $T_{\alpha}(A)$ be the same as in Example 1. Then, elements of $\mathscr{C}_{1}$ are elementary cycles and $\alpha$-revived cycles in Example 1 . We have card $\left(\mathscr{C}_{1} / \equiv\right)=1$. 
On the other hand, $\mathscr{A}_{1}=\left\{x_{4}, z_{3}, y_{z_{1} z_{2}}, y_{x_{1} z_{2}}\right\}$. We have

$$
\begin{aligned}
& x_{4} z_{1} \neq 0, \quad z_{3} z_{1} \neq 0, \quad y_{z_{1} z_{2}} z_{1} \neq 0, \\
& x_{4} x_{1} \neq 0, \quad y_{x_{1} z_{2}} x_{1} \neq 0,
\end{aligned}
$$

in $T_{\alpha}(A)$. So all elements in $\mathscr{A}_{1}$ are equivalent, and $\operatorname{card}\left(\mathscr{A}_{h} / \approx\right)=1$. So, for almost split sequence

$$
0 \longrightarrow S_{1} \longrightarrow E \longrightarrow \tau^{-1} S_{1} \longrightarrow 0,
$$

$E$ is a non-projective indecomposable module by Theorem 4.4.

Example 3. Let $\Delta$ be the following quiver:

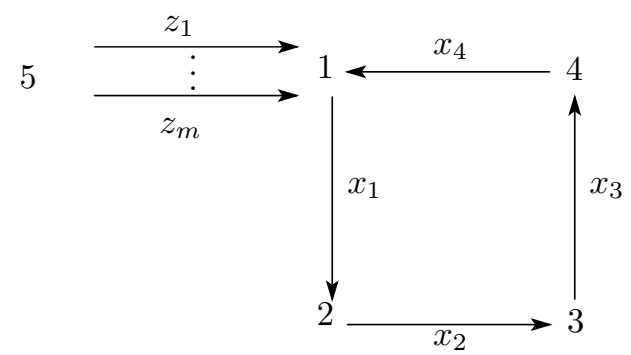

and $A=K \Delta / R_{\Delta}^{3}$. We put $\gamma=x_{1} x_{2} x_{3} x_{4}$. Then, for each $i(1 \leq i \leq 4), \alpha_{i}$ : $A \times A \longrightarrow D(A)$ corresponding to $\gamma$ is the map as follows:

$$
\alpha_{i}(\bar{a}, \bar{b})= \begin{cases}{\overline{x_{i+3}}}^{*} & \text { if } \bar{a}, \bar{b} \neq 0 \text { in } A \text { and } a b=x_{i} x_{i+1} x_{i+2}, \\ \bar{e}_{i+4} & \text { if } \bar{a}, \bar{b} \neq 0 \text { in } A \text { and } a b=x_{i} x_{i+1} x_{i+2} x_{i+3} \\ 0 & \text { otherwise, }\end{cases}
$$

where the definition of $\alpha_{i}$ 's is given in Section 2. Let $k(\neq 0) \in K$ and $\alpha=$ $k \sum_{i=1}^{4} \alpha_{i}$. Then, the ordinary quiver of $T_{\alpha}(A)$ coincides with $\Delta_{T_{0}(A)}$, and $\Delta_{T_{0}(A)}$ is the following quiver:

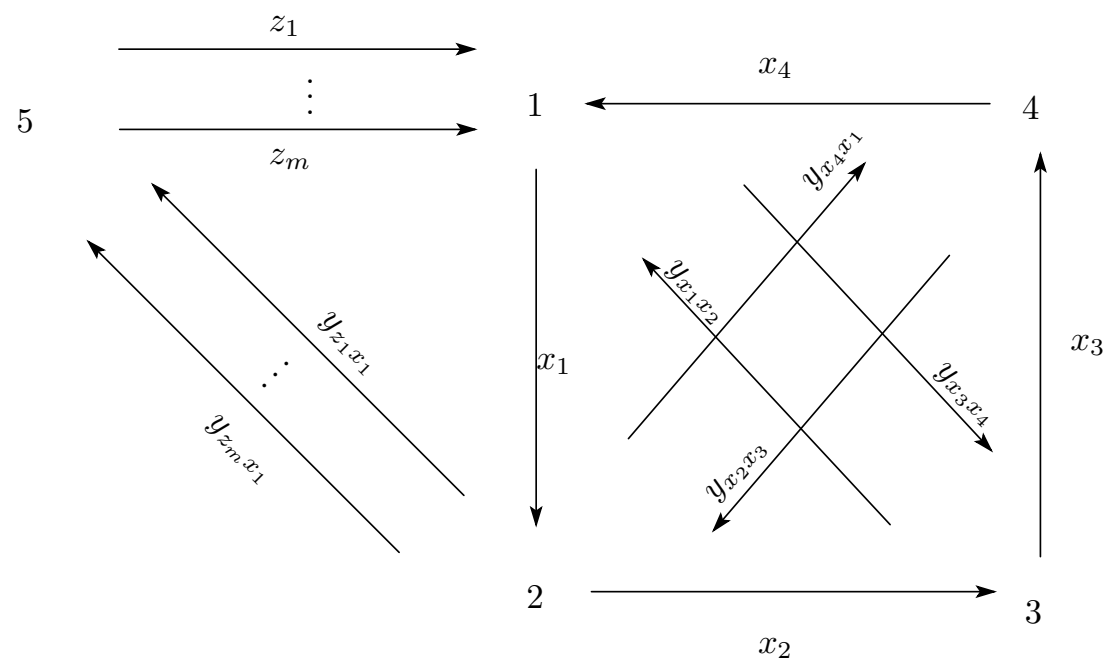

In this case, elementary cycles with origin 5 are

$$
z_{1} x_{1} y_{z_{1} x_{1}}, z_{2} x_{1} y_{z_{2} x_{1}}, \ldots, z_{m} x_{1} y_{z_{m} x_{1}} \text {. }
$$


Moreover, there is no $\alpha$-revived cycle with origin 5. In this case, if $i \neq j$ then $z_{i} x_{1} y_{z_{i} x_{1}}$ and $z_{j}^{\prime} x_{1} y_{z_{j}^{\prime} x_{1}}$ are not equivalent in $\mathscr{C}_{5}$. And we have $\operatorname{card}\left(\mathscr{C}_{5} / \equiv\right)=m$.

\section{ACKNOWLEDGMENT}

The author is grateful to Professor Katsunori Sanada and Doctor Tomohiro Itagaki for many helpful comments and suggestions on improving the clarity of the article.

\section{REFERENCES}

[1] I. Assem, D. Simson, A. Skowroński, Elements of the Representation Theory of Associative Algebras I, London Math. Soc. Student Texts, vol. 65. Cambridge: Cambridge Univ. Press, 2006.

[2] S. Brenner, The almost split sequence starting with a simple module, Arch. Math. 62 (1994) 203-206.

[3] E. Fernández, M. Platzeck, Presentations of trivial extensions of finite dimensional algebras and a theorem of Sheila Brenner, J. Algebra 249 (2002) 326-344.

[4] G. Hochschild, On the cohomology groups of an associative algebra, Ann. Math. (2) 46 (1945) $58-67$.

[5] H. Koie, T. Itagaki, K. Sanada, The ordinary quivers of Hochschild extension algebras for self-injective Nakayama algebras, Communications in Algebra, 46 (2018) No.9, 3950-3964.

[6] H. Koie, T. Itagaki, K. Sanada, On presentations of Hochschild extension algebras for a class of self-injective Nakayama algebras, SUT Journal of Mathematics 53 (2017) No.2, 135-148.

[7] E. Sköldberg, The Hochschild homology of truncated and quadratic monomial algebra, J. Lond. Math. Soc. (2) 59 (1999) 76-86.

[8] A. Skowroński, K. Yamagata, Frobenius Algebras I: Basic Representation Theory, EMS Textbooks Math. Zürich: Eur. Math. Soc. 2011.

[9] K. Yamagata, Extension over hereditary artinian rings with self-dualites, I, J. algebra $\mathbf{7 3}$ (1981) 386-433.

[10] K. Yamagata, Frobenius Algebras, Handbook of Algebra 1: 841-887, Elsevier/ North-Holland, Amsterdam, 1996.

(H. Koie) Department of Mathematics, Tokyo University of Science, 1-3 Kagurazaka, SHINJUKU-KU, TOKYO 162-8601, JAPAN

E-mail address: 1114702@ed.tus.ac.jp 